\title{
Ghrelin Attenuated Lipotoxicity via Autophagy Induction and Nuclear Factor- KB Inhibition
}

\author{
Yuqing Mao ${ }^{a}$ Jian Cheng ${ }^{a}$ Fujun Yu ${ }^{a}$ Huanqing $\mathrm{Li}^{\mathrm{a}}$ Chuanyong Guo ${ }^{\mathrm{b}}$ Xiaoming Fan ${ }^{\mathrm{a}}$ \\ aDepartment of Gastroenterology and Hepatology, Jinshan Hospital of Fudan University, Shanghai, \\ ${ }^{b}$ Department of Gastroenterology and Hepatology, Shanghai Tenth People's Hospital of Tongji \\ University, Shanghai, China
}

\section{Key Words}

Nonalcoholic fatty liver disease (NAFLD) • Free fatty acid (FFA) • High fat diet (HFD) • Autophagy - Ghrelin • Nuclear factor-кB

\begin{abstract}
Background/Aims: Nonalcoholic fatty liver disease (NAFLD) is the most common chronic liver disease worldwide. Autophagy is associated with NAFLD. Ghrelin is a gut hormone with various functions including energy metabolism and inflammation inhibition. We investigated the therapeutic effect of ghrelin on NAFLD and its association with autophagy. Methods: C57bl/6 mice were fed a high-fat diet for 8 weeks to induce a model of chronic NAFLD, with ghrelin $(10 \mu \mathrm{g} / \mathrm{kg})$ administrated subcutaneously twice weekly from weeks 6 to 8. LO2 cells were pretreated with ghrelin $\left(10^{-8} \mathrm{M}\right)$ before stimulation with free fatty acid (palmitic and oleic acids; $1 \mathrm{mM}$ ). Lipid droplets were identified by hematoxylin and eosin and Red $\mathrm{O}$ staining and quantified by triglyceride test kits. LC3I/II, an important biomarker protein of autophagy was detected by western blotting, real-time polymerase chain reaction, immunohistochemistry and immunofluorescence. Tumor necrosis factor (TNF)- $\alpha$ and interleukin (IL)- 6 were detected by ELISA and immunohistochemistry. Nuclear factor (NF)-kB p65 was detected by western blotting and immunofluorescence. AMP-activated protein kinase (AMPK) and mammalian target of rapamycin (mTOR) were detected by western blotting. Results: Ghrelin reduced the triglyceride content in high fat diet (HFD) group in vivo and free fatty acid (FFA) group in vitro. TNF- $\alpha$ and IL- 6 were significantly reduced in the ghrelin-treated mice compared with the control group. Autophagy induction was accompanied with intracellular lipid reduction in ghrelin-treated mice. Ghrelin upregulated autophagy via AMPK/mTOR restoration and inhibited translocation of NF-KB into the nucleus. Conclusions: The results indicate that ghrelin attenuates lipotoxicity by autophagy stimulation and NF-KB inhibition.
\end{abstract}

Professor Chuanyong Guo, MD\&PhD and Professor Xiaoming Fan, MD\&PhD

\section{KARGER 125}

Department of Gastroenterology and Hepatology, Shanghai Tenth People's Hospital, Tongji University, Shanghai 200072, (China); and Department of Gastroenterology and Hepatology, Jinshan Hospital of Fudan University, Shanghai 201508, (China)

E-Mail guochuanyong@hotmail.com and E-Mail xiaomingfan57@hotmail.com 


\section{Cellular Physiology Cell Physiol Biochem 2015;37:563-576 \\ \begin{tabular}{l|l|l} 
DOI: 10.1159/000430377 & C 2015 S. Karger AG, Basel
\end{tabular} \\ and Biochemistry Published online: September 01, $2015 \quad$ www.karger.com/cpb \\ Mao et al.: Ghrelin Reduced Lipotoxicity}

\section{Introduction}

Nonalcoholic fatty liver disease (NAFLD) is the most common chronic liver disease worldwide. The prevalence of NAFLD in adults in the US is currently estimated at $\leq 46 \%$ [1], which is of major concern, with its progression from steatosis to steatohepatitis, cirrhosis, and even hepatocellular carcinoma. At present, there are no effective drug therapies for NAFLD. According to the classical "two hits" theory [2], both insulin resistance and oxidative stress contribute to initiation and progression of NAFLD. Increased lipid is not offset by mitochondrial oxidation or by increased secretion of lipoproteins[3].As a result, oxidative stress occurs, causing hepatocyte injury with reactive oxygen species (ROS), which lead to cell inflammation and even apoptosis, called lipoapoptosis [4]. Moreover, a recent study showed there is a critical role for miRNAs in the pathogenesis of NAFLD [5].

On the way from fat to inflammation, nuclear factor (NF)- $\kappa B$ signaling pathway activation is characterized as the most important mechanism, which functions as a proinflammatory "master switch" by upregulating transcription of a wide range of inflammatory mediators [6]. Lipid accumulation induced by high-fat diet (HFD) in the liver of mice leads to subacute hepatic inflammation via NF- $\kappa$ B activation, with increased downstream cytokines such as tumor necrosis factor (TNF)- $\alpha$, interleukin (IL)-6 and IL-1 $\beta$ [7].

Autophagy is an intracellular process of self-digesting excess or defective organelles to maintain homeostasis, which has entered the research spotlight due to its wide involvement in different diseases. Autophagy mainly acts as a cell survival mechanism. However, excessive autophagy may also contribute to cell death called type II programmed cell death, suggesting that autophagy is a double-edged sword [8]. The new function of autophagy in regulating intracellular lipid stores is called macrolipophagy, which is regarded as liver-specific autophagy. The pharmacological inhibition of autophagy with 3-methyladenine (3-MA) and autophagy-related gene (Atg) knock down increase intracellular lipid storage [9]. Ablation of macrolipophagy leads to excessive hepatic lipid accumulation and development of NAFLD [10]. The latest research has discovered that caffeine stimulates hepatic lipid mechanism and increases hepatic fat clearance via the autophagy-lysosome pathway [11], which makes us more confident that autophagy is a new therapeutic target for NAFLD.

Ghrelin is a novel peptide that was first purified from the stomach, and is the endogenous ligand for growth hormone secretagogue receptor [12]. It is a brain-gut peptide existing in two forms, with the octanoyl form regarded as the active form. Circulating ghrelin is mostly produced in X/A-like cells of the stomach and small intestine. However, small amounts of ghrelin are also produced in other organs such as pancreas, lung, kidney and pituitary, and adipocyte tissue. Ghrelin is a multifunctional peptide showing roles in orexigen, energy regulation, and growth hormone release. Recently, ghrelin was demonstrated to improve inflammatory reactions by enhancing immune-cell proliferation and inhibiting secretion of proinflammatory cytokines [13]. Li et al. first demonstrated that ghrelin administration in rats attenuated NAFLD-induced hepatic injury, inflammation and apoptosis, partly through restoration of LKB1/AMPK and PI3K/Akt pathways [14], indicating that ghrelin is a new treatment for NAFLD.

However, the mechanisms of ghrelin-regulated lipid metabolism are not clear. Research in rodent models of NAFLD is limited. In some studies, ghrelin was reported to improve cardiac cell injury by inducing autophagy [15] or inhibiting excessive autophagy [16]. However, whether ghrelin is associated with autophagy in ameliorating NAFLD-induced liver injury has not been investigated. The study was designed to investigate whether ghrelin reduced hepatic triglyceride and inflammation injury in NAFLD, with or without participation of autophagy.

\section{Materials and Methods}

Reagents

Ghrelin was purchased from Prospec (Israel). HFD (D12492), composed of 20\% protein, 60\% fat, and $20 \%$ carbohydrate of the caloric intake; $5.24 \mathrm{kcal} / \mathrm{g}$ was from Shanghai QF Biosciences (Shanghai, China). 


\section{Cellular Physiology Cell Physiol Biochem 2015;37:563-576 \\ \begin{tabular}{l|l|l}
\hline DOI: 10.1159/000430377 & (C) 2015 S. Karger AG, Basel
\end{tabular} \\ and Biochemistry Published online: September 01, 2015 \\ www.karger.com/cpb \\ Mao et al.: Ghrelin Reduced Lipotoxicity}

The following chemicals were all from Sigma-Aldrich (St Louis, MO, USA): bovine serum albumin (BSA), oleic acid (OA), palmitic acid (PA), 3-methyladenine (3-MA), and rapamycin. Antibodies against NF- $\kappa \mathrm{B}$ p65 were purchased from Abcam (Cambridge, MA, USA). The following antibodies were all purchased from Cell Signaling Technology (Danvers, MA, USA): LC3, Beclin 1, IKB $\alpha$, phosphorylated AMPK (p-AMPK) at Thr172, total AMPK, phosphorylated mTOR(p-mTOR), total mTOR and lamin-A.

\section{Cell culture and treatment}

Normal human liver cell line LO2 was purchased from the Chinese Academy of Science Committee Type Culture Collection Cell Bank. Cells were cultured in high-glucose Dulbecco's modified Eagle's medium (Gibco, USA) supplemented with 10\% fetal bovine serum (Gibco, South America) and 1\% penicillinstreptomycin (Gibco, USA) in a humidified incubator at $37^{\circ} \mathrm{C}$ in $5 \% \mathrm{CO}_{2}$. OA and PA (20 mM solution) were dissolved in $5 \% \mathrm{BSA}$ which had been previously heated at $37^{\circ} \mathrm{C}$ in a water bath for several hours. LO2 cells were exposed to $1 \mathrm{mM}$ free fatty acid (FFA) mixture (OA: PA, 2:1) for $24 \mathrm{~h}$ to induce steatosis. Cells were pretreated with ghrelin at concertration of $10 \mathrm{nM}$ for $1 \mathrm{~h}$ before stimulation with FFA. Cells were divided into four groups: 1. Normal control group (NC): treated with PBS only as vehicle; 2 . NC+ ghrelin group: treated with ghrelin diluted in PBS at a concentration of $10^{-8} \mathrm{M}$; 3. FFA group: treated with $1 \mathrm{mM}$ FFA mixture for 24 $\mathrm{h}$; and 4. FFA+ ghrelin group: treated with ghrelin and FFA mixture described above.

Animals and treatment

C57BL/6 mice (8 weeks old, $23 \pm 2$ g) were purchased from Shanghai Laboratory Animal Co. Ltd. (Shanghai, China). The mice were housed in a clean room maintained at $24 \pm 2{ }^{\circ} \mathrm{C}$ with an alternating 12 -h light and dark cycle, with free access to food and water. All animal experiments were approved by the Animal Care and Use Committee of Shanghai Fudan University. Mice were randomly divided into four groups with 7 mice each group. 1. Saline group: mice were fed with normal rat diet with saline injected intraperitoneally daily from week 6 to 8. 2. Saline+ ghrelin group: mice were fed with normal rat diet for 8 weeks, with ghrelin injected intraperitoneally daily from week 6 to 8 at a dose of $10 \mu \mathrm{g} / \mathrm{kg}$. 3. HFD group: mice were fed with HFD for 8 weeks. 4. HFD+ ghrelin group: mice were fed with HFD for 8 weeks with ghrelin injected intraperitoneally twice weekly from week 6 to 8 at a dose of $10 \mu \mathrm{g} / \mathrm{kg}$. All mice were sacrificed at $8^{\text {th }}$ week. Liver samples and blood were acquired for histological and molecular analysis.

\section{Biochemical assays}

The serum levels of ALT and AST were measured with microplate test kits (Nanjing Jiancheng Bioengineering Institute, China). Serum proinflammatory cytokines TNF- $\alpha$ and IL- 6 were measured by ELISA kits (R\&D Systems, Minneapolis, MN, USA). Triglyceride levels in LO2 cells were measured with a microplate test kit from Nanjing Jiancheng Bioengineering Institute.

\section{Histopathology}

The middle portion of the left lobe of the liver of each mouse was excised and sectioned and then perfused in $4 \%$ paraformaldehyde for at least $24 \mathrm{~h}$. After fixation, the tissues were embedded in paraffin, and $5-\mu \mathrm{m}$ thick sections were stained with hematoxylin and eosin (H\&E) to observe the tissue damage by light microscopy.

\section{Immunohistochemistry}

The liver sections $(3 \mu \mathrm{m})$ were dewaxed and rehydrated regularly and treated with $3 \% \mathrm{H}_{2} \mathrm{O}_{2}$. The sections were pretreated with a microwave antigen retrieval technique. The nonspecific sites were blocked with $10 \%$ goat serum for $30 \mathrm{~min}$ at room temperature. The liver slices were incubated overnight with antibody directed against Beclin-1 (1:500) and LC3II (1:500). On the second day, after incubation with secondary antibody, an antibody binding assay was performed using a diaminobenzidine kit. Three different fields of vision were randomly selected in one slide, and the ratios of positive areas to total areas were acquired with Image-Pro Plus 6.0. We calculated the average of these three ratios. The above method was applied in all groups.

\section{Western blotting}

Liver tissues and LO2 cells were collected and lysed with RIPA buffer and protease inhibitor. The protein concentrations were measured with the BCA method. Equivalent amounts of total protein were separated by SDS-PAGE and transferred to PVDF membranes. Nonspecific binding was blocked with 5\% 


\section{Cellular Physiology Cell Physiol Biochem 2015;37:563-576 \\ \begin{tabular}{l|l|l}
\hline DOI: 10.1159/000430377 & C 2015 S. Karger AG, Basel
\end{tabular} \\ and Biochemistry Published online: September 01, 2015 \\ www.karger.com/cpb \\ Mao et al.: Ghrelin Reduced Lipotoxicity}

nonfat milk (dissolved in PBS) for $1 \mathrm{~h}$ and then incubated overnight at $4^{\circ} \mathrm{C}$ with antibodies directed against LC3II (1:500), Beclin-1 (1:1000), NF-кB p65(1:500), IкB (1:500), pAMPK (1:500), total AMPK (1:500), $\beta$-actin (1:1000),p-mTOR(1:500), mTOR(1:500) and lamin-A (1:500). All membranes were washed with PBS $+1 \%$ Tween (PBST) and incubated with a secondary antibody (1:1000) dissolved in PBST, for $1 \mathrm{~h}$ at $37^{\circ} \mathrm{C}$. Finally, the membranes were washed three times with PBST for $10 \mathrm{~min}$ and the proteins were detected with the Odyssey two-color infrared laser imaging system (detected with fluorescence).

Oil Red 0 staining

LO2 cells were cultivated in six-well plates, processed with the relevant treatment, rinsed three times with PBS, fixed in 4\% paraformaldehyde for $30 \mathrm{~min}$, stained for $15 \mathrm{~min}$ at room temperature in freshly diluted Oil Red O, and rinsed twice with PBS. Finally, intracellular lipid droplets were observed with a light microscope.

\section{Immunofluorescence staining}

Immunofluorescence staining was performed according to standard protocols. The LO2 cells were washed three times with PBS solution for $1 \mathrm{~min}$. The cells were fixed with 4\% paraformaldehyde for 30 min and washed three times with PBS for 5 min. Nonspecific antigen-binding sites were blocked with $5 \%$ BSA and cells were incubated overnight with anti-LC3II antibody (1:50) at $4{ }^{\circ} \mathrm{C}$. On the second day, cells were washed three times with PBS for $5 \mathrm{~min}$ and incubated with anti-rabbit antibody for $30 \mathrm{~min}$. 2-(4-amidinophenyl)-6-indolecarbamidine dihydrochloride (DAPI) (Life Technologies, USA) was used to stain cell nuclei. Tandem GFP-tagged LC3 virus (Invitrogen, Carlsbad, CA, USA) was used to infect cells for $24 \mathrm{~h}$. All images were acquired using ZEN software (Carl Zeiss AG, Germany).

RNA isolation and real-time quantitative reverse transcriptase polymerase chain reaction ( $q R T-P C R$ )

The liver tissue and LO2 cells were detected and analyzed with qRT-PCR. Total RNA was extracted from frozen liver tissues or cells with TRIzol reagent (Tiangen Biotech, China). SYBR Green Quantitative RT-PCR was performed with the 7900HT Fast Real-Time PCR system (Applied Biosystems, CA, USA) to determine expression of the target genes, according to the instructions for SYBR Premix EX Taq (TaKaRa Biotechnology, China).The primer sequences are shown in Table 1.

\section{Electron microscopy}

Mice and LO2 cells were treated as described above, and fresh liver tissue was perfused with $2 \mathrm{~mL} \mathrm{2.5 \%}$ glutaraldehyde in PBS. LO2 cells were collected from six-well plates and perfused with glutaraldehyde as described above. The tissue and cells were sectioned and examined under a transmission electron microscope (Tecnai) at $160 \mathrm{kV}$. Electron micrographs were obtained using Electron Microscopy Film 4489 (Kodak, ESTAR thick base) and printed onto photographic paper.

\section{Statistical analysis}

All results are expressed as means \pm SD. Comparisons between two groups were made with Student's $t$ test. Statistical differences in multiple groups were determined by multiple comparisons with analysis of variance, followed by Tukey's post hoc test. All statistical analyses were performed with Graphpad Prism version 6.0 software. $P<0.05$ was considered statistically significant.

Table 1. Nucleotide sequences of primers used for PCR

\begin{tabular}{lll}
\hline Gene & & Primer sequence (5'-3') \\
\hline LC3 & Forward & TGCTGTCCCGAATGTCTCCTG \\
& Reverse & GCTAACCAAGCCTTCTTCCTCC \\
Beclin-1 & Forward & AGATGCCTCCCCGATCAGAG \\
& Reverse & CTTACCACAGCCCAGGCGAA \\
B-Actin & Forward & GGCTGTATTCCCCTCCATCG \\
& Reverse & CCAGTTGGTAACAATGCCATGT \\
\hline
\end{tabular}




\begin{tabular}{|c|c|c|}
\hline Cellular Physiology & Cell Physiol Biochem 2015;37:563-576 & \\
\hline and Biochemistry & $\begin{array}{l}\text { DOI: 10.1159/000430377 } \\
\text { Published online: September 01, } 2015\end{array}$ & $\begin{array}{l}\text { (0) } 2015 \text { S. Karger AG, Basel } \\
\text { www.karger.com/cpb }\end{array}$ \\
\hline
\end{tabular}

\section{Results}

Ghrelin administration reduced lipid accumulation

Oil Red 0 staining of cells showed ghrelin pretreatment before FFA stimulation significantly reduced intracellular lipid accumulation, which was quantified by triglyceride test kit (Fig. 1A and 1B). The results were confirmed using H\&E staining (Fig. 1D) and Oil Red 0 staining (Fig. 1E) of mice liver tissues. The liver was photographed and liver weight/ body weight ratio was calculated. Actually we used two doses of ghrelin to treat HFD mice $(10 \mathrm{ng} / \mathrm{kg}$ and $10 \mu \mathrm{g} / \mathrm{kg}$ ) in the preliminary experiment and found high dose of ghrelin group showed better effect. Therefore, we used $10 \mu \mathrm{g} / \mathrm{kg}$ ghrelin in the following experiment. The HFD mice had hepatomegaly due to swollen hepatocytes and more shallow hepatic color visually, while the liver tissue in the ghrelin co-treatment group was nearly normal size and fresh red visually especially the high dose of ghrelin group. HFD led to a higher liver/body weight ratio compared with that in the saline group, which was significantly reduced by $10 \mu \mathrm{g} / \mathrm{kg}$ ghrelin treatment, as well as the indicators of liver triglyceride level and serum cholesterol level (Fig. 1C).

Ghrelin administration attenuated hepatic inflammation induced by lipotoxicity

As indicators of liver injury, elevated serum levels of ALT and AST were observed in HFD group, which were significantly reduced by co-treatment with ghrelin (Fig. 2A). Serum levels of pro-inflammatory cytokines (TNF- $\alpha$ and IL-6) were significantly upregulated in HFD mice and down-regulated in the ghrelin+ HFD group (Fig. 2B). Immunohistochemical staining showed ghrelin significantly reduced the expression of TNF- $\alpha$ and IL- 6 compared with HFD group (Fig. 2C and 2D). There were no significant differences of hepatic enzymes or proinflammatory cytokines between the supernatant of cultured LO2 cells from the NC, FFA and $\mathrm{FFA}+$ ghrelin groups (data not shown).

Ghrelin-induced lipid clearance was associated with a concomitant increase in autophagy

Five methods were used to examine the relationship between lipid clearance and autophagyincludingwestern blotting, qRT-PCR,immunohistochemistry,immunofluorescence and electron microscopy. Increased protein levels of LC3 and Beclin-1 were observed in the HFD or FFA group, which were further stimulated by ghrelin co-treatment in vivo and in vitro (Fig. 3 and 4). We investigated mRNA transcription level of LC3 and Beclin-1and found similar results. Protein levels of LC3II and Beclin-1 were also measured by immunohistochemistry (Fig. 4B). Immunofluorescence of LC3II showed that autophagic flux was increased in HFD group and FFA group, while ghrelin treatment further activate it both in vitro and in vivo (Fig. 3B and Fig. 4C). Similarly, adenovirus carrying GFP-LC3 detected increased expression of LC3II in ghrelin co-treated group (green dots in merged images shown in Fig. 3C). Electron microscopy showed increased autophagosomes in the ghrelin co-treated group in vivo and in vitro (Fig. 3D and Fig. 4D).

\section{Autophagy inhibition reversed reduction of intracellular lipid droplets}

To investigate whether the ghrelin-induced reduction in intracellular triglyceride was reversed by autophagy inhibition, $10 \mathrm{mM} 3$-MA (autophagy inhibitor) and $1 \mu \mathrm{M}$ rapamycin (autophagy enhancer) were used to treat LO2 cells. Reduced level of LC3II was detected in the 3-MA+ ghrelin+ FFA group compared with the FFA+ ghrelin group. On the contrary, increased level of LC3II was observed in the rapamycin+ ghrelin+ FFA group, indicating that the ghrelin-induced increase in autophagy was inhibited by 3-MA and augmented by rapamycin stimulation. The intracellular lipid level in the 3-MA+ghrelin+ FFA group was higher than that in the ghrelin+ FFA group, while rapamycin treatment significantly reduced intracellular lipid accumulation of steatotic LO2 cells (Fig. 3E).

Ghrelin promoted autophagy partly via restoration of the AMPK/mTOR signaling pathway

HFD or FFA dramatically reduced phosphorylation of AMPK (pAMPK) while ghrelin restored it without affecting the level of total AMPK. As shown in Fig. 5, mTOR, an AMPK 


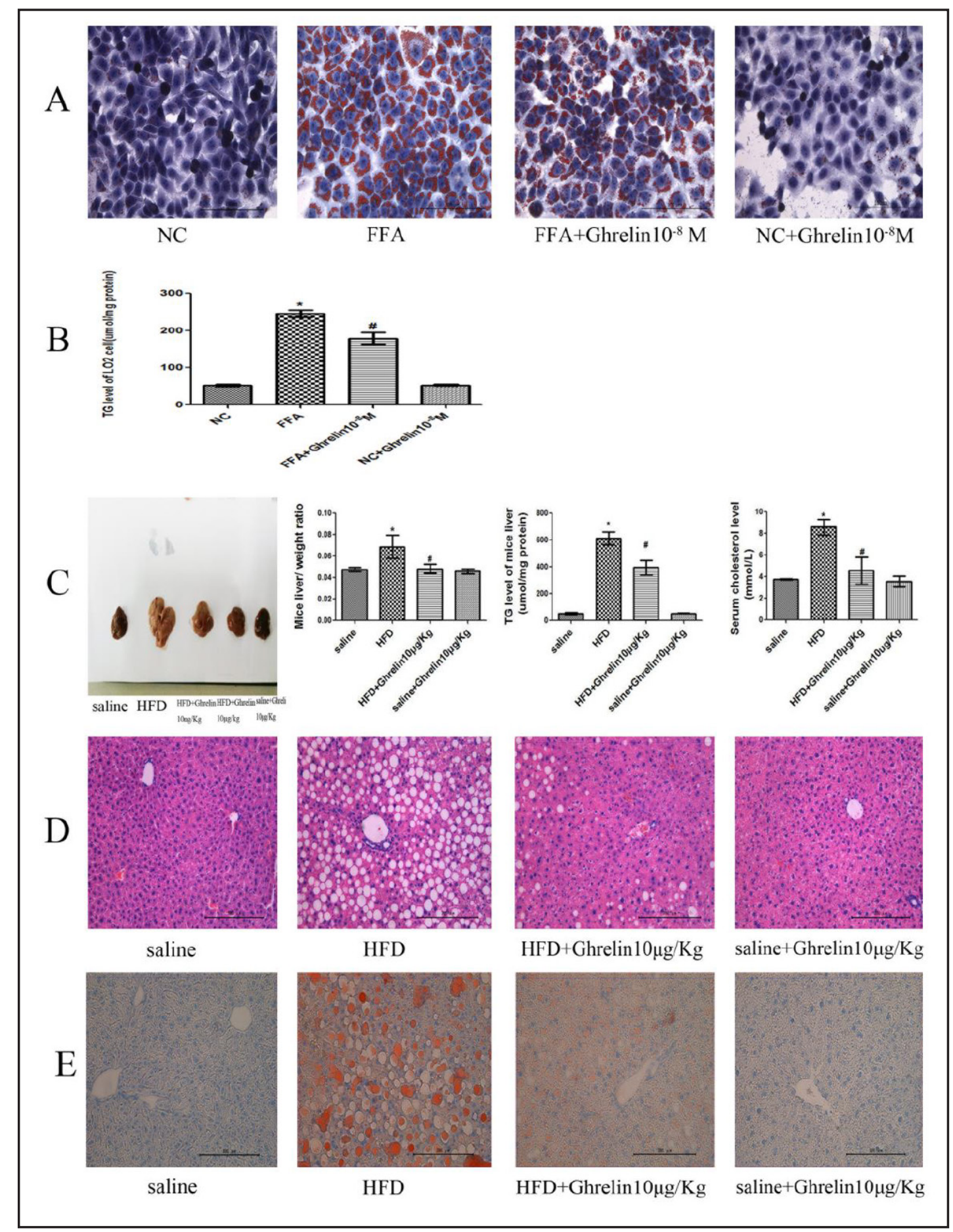

Fig. 1. Ghrelin reduced lipid accumulation in vitro and in vivo. (A) Representative images of LO2 cells stained with Oil Red 0, which were exposed to $1 \mathrm{mM}$ FFA mixture (OA and PA; 2:1) and ghrelin (10-8 M) for $24 \mathrm{~h}$, compared to FFA-treated cells, NC group and NC+ ghrelin group. (B) Intracellular triglyceride levels were quantified in the four groups. ${ }^{*} P<0.05$ for FFA group versus NC group; $\# P<0.05$ for FFA+ ghrelin group versus FFA group. (C) Liver from mice groups was photographed: saline group; HFD group; HFD+ ghrelin group (low-dose ghrelin $10 \mathrm{ng} / \mathrm{kg}$, high-dose ghrelin $10 \mu \mathrm{g} / \mathrm{kg}$ ). Mouse liver/body weight ratio, liver triglyceride content, and serum cholesterol level were detected. ${ }^{*} P<0.05$ for HFD group versus saline group; $\# P<0.05$ for HFD+ ghrelin group versus HFD group. (D) H\&E staining of mouse liver sections from the four groups described above. (E) Representative images of liver sections stained with Oil Red $\mathrm{O}$ from the above groups. 


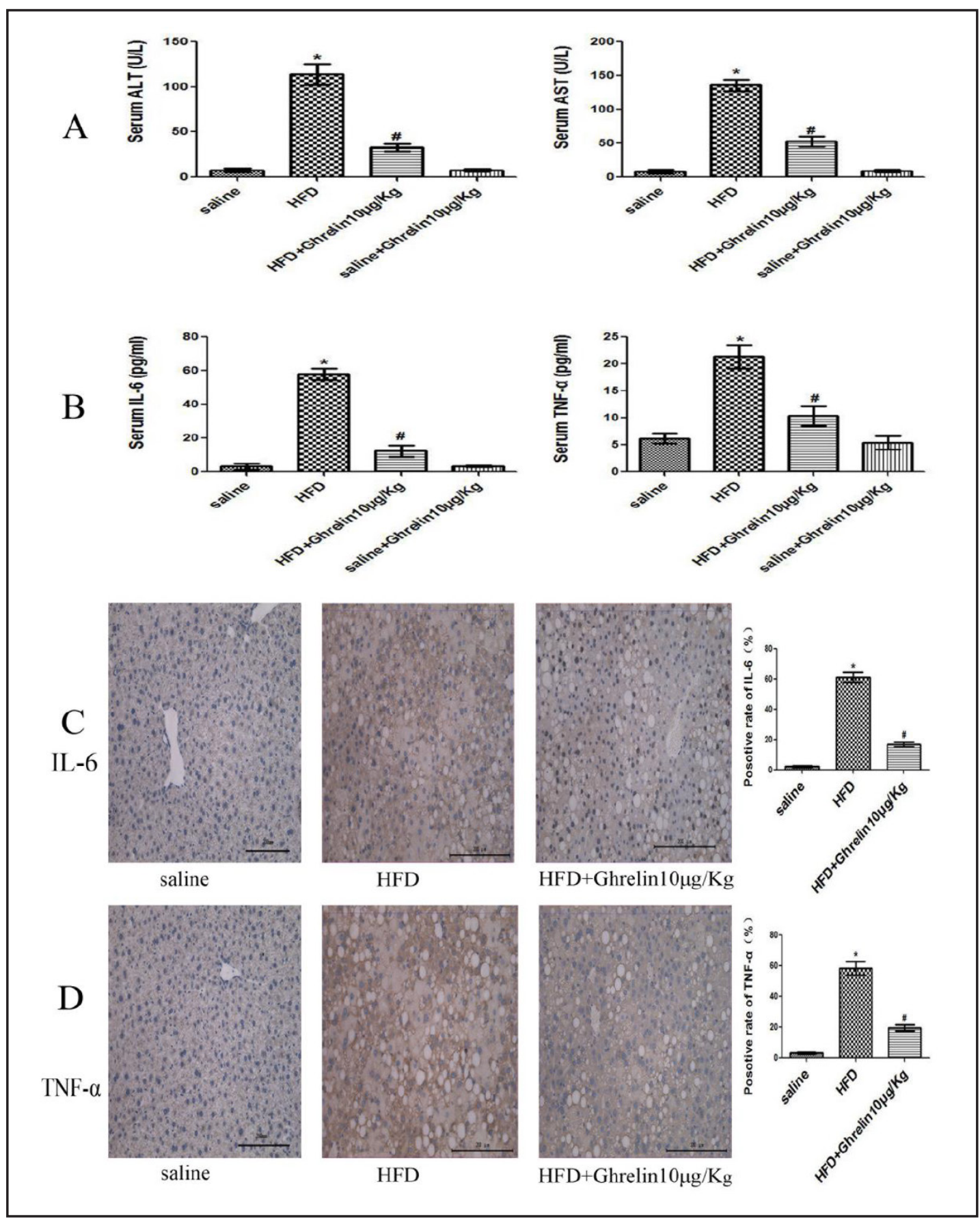

Fig. 2. Ghrelin administration attenuated hepatic inflammation induced by lipotoxicity. (A)Serum level of serum ALT and AST level were detected by ELISA in mice groups with or without ghrelin co-treatment. (B) Serum level of pro-inflammatory cytokines IL- 6 and TNF- $\alpha$ were detected by ELISA in mice groups with or without ghrelin co-treatment. (C and D) Immunohistochemistry staining $(200 \times)$ showed protein level of TNF- $\alpha$ and IL- 6 in liver tissue of mice after NAFLD induction, with or without ghrelin co-treatment. ${ }^{*} P<0.05$ for HFD group versus saline group; $\# P<0.05$ for HFD+ ghrelin group versus HFD group.

downstream and its phosphorylation form (p-mTOR) were detected in mice groups. Ghrelin treatment inhibited mTOR phosphorylation in vivo and in vitro. We demonstrated that ghrelin attenuated lipotoxicity by enhancing autophagy partly via restoration of the AMPK/ mTOR signaling pathway.

\section{KARGER}




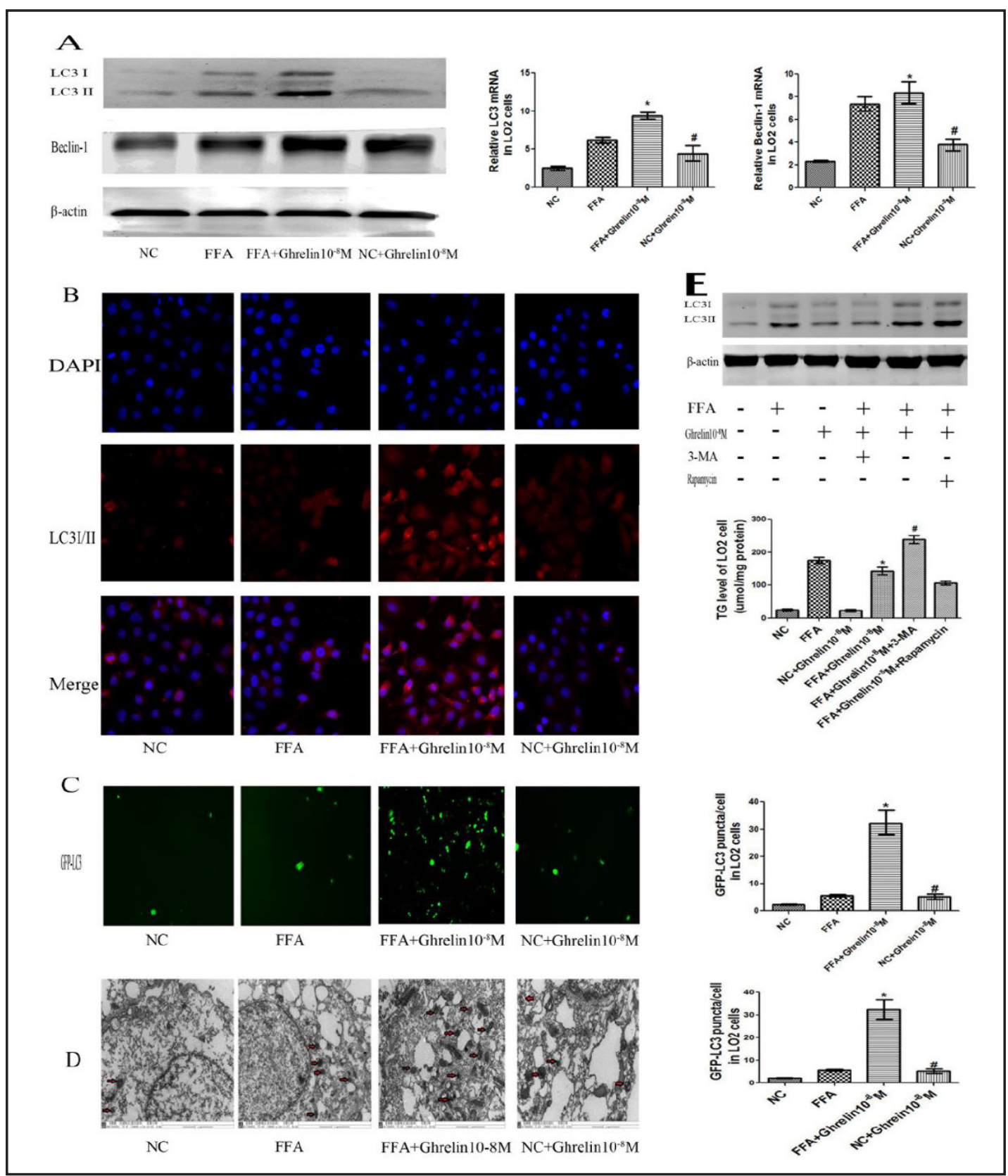

Fig. 3. Ghrelin-induced lipid clearance is associated with a concomitant increase in autophagy in LO2 cells. (A) Protein level of LC3I/II and Beclin-1were detected by western blotting and mRNA expression of LC3I/II and Beclin- 1 was detected by real-time qRT-PCR. ${ }^{*} P<0.05$ for FFA+ ghrelin group versus FFA group; $\# P<0.05$ for NC+ ghrelin group versus NC group. (B) LC3 immunostaining showed increased endogenous LC3-II level in LO2 cells, which were pretreated with ghrelin (10-8 M) before stimulation with FFA, compared with cells treated with FFA alone, NC group and NC+ ghrelin group. (C) Adenovirus carrying GFP-LC3 detected the expression of LC3II in vitro. The average number of autophagosomes/cell \pm SD counted from fluorescence microscopy images of LO2 cells expressing GFP-LC3 (green dots in merged images shown in Fig.3C, Original magnifications: $\times 400$ ). ${ }^{*} P<0.05$ for FFA+ ghrelin group versus FFA group; $\# P<0.05$ for NC+ ghrelin group versus NC group. (D) Electron microscopy showed the ultrastructure and autophagosomes (indicated by $\rightarrow$ ). (Original magnifications: $\times 10000$ ). (E)Protein level of LC3I/II was detected by western blotting in the groups treated with or without autophagy inhibitor 3-MA or stimulator rapamycin in LO2 cells. Intracellular triglyceride levels were detected. ${ }^{*} P<0.05$ for FFA+ ghrelin group versus FFA group; $\# P<0.05$ for FFA + ghrelin+3-MA group versus FFA+ ghrelin group.

\section{KARGER}




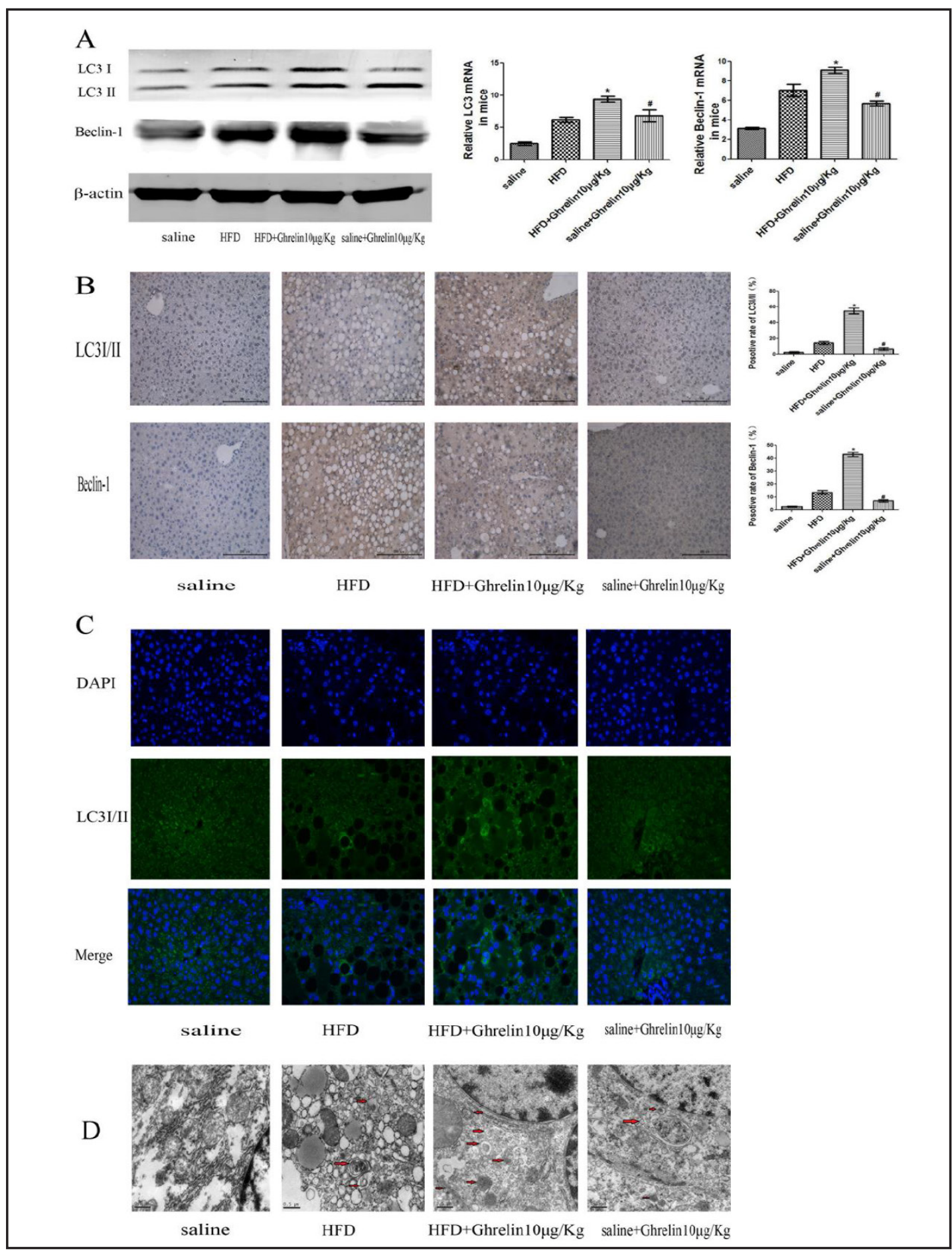

Fig. 4. Ghrelin-induced lipid clearance was associated with a concomitant increase in autophagy in mice. (A) Protein level of LC3I/II and Beclin-1 in mice was detected by western blotting and mRNA expression of LC3I/II and Beclin- 1 was detected by real-time qRT-PCR. ${ }^{*}<<0.05$ for HFD+ ghrelin group versus HFD group; $\# P<0.05$ for saline+ ghrelin group versus saline group. (B) Immunohistochemistry staining $(200 \times)$ showed level of LC3I/II and Beclin-1 in liver tissue. ${ }^{*} P<0.05$ for HFD+ ghrelin group versus HFD group; $\# P<0.05$ for saline+ ghrelin group versus saline group. (C) LC3 immunostaining of mice liver tissues showed increased endogenous LC3-II level in HFD+ ghrelin mice group, compared with HFD group, normal group and saline+ ghrelin group. (D) Electron microscopy showed ultrastructure and autophagosomes (indicated by $\rightarrow$ ). (Original magnifications: $\times 20000$ ).

\section{KARGER}




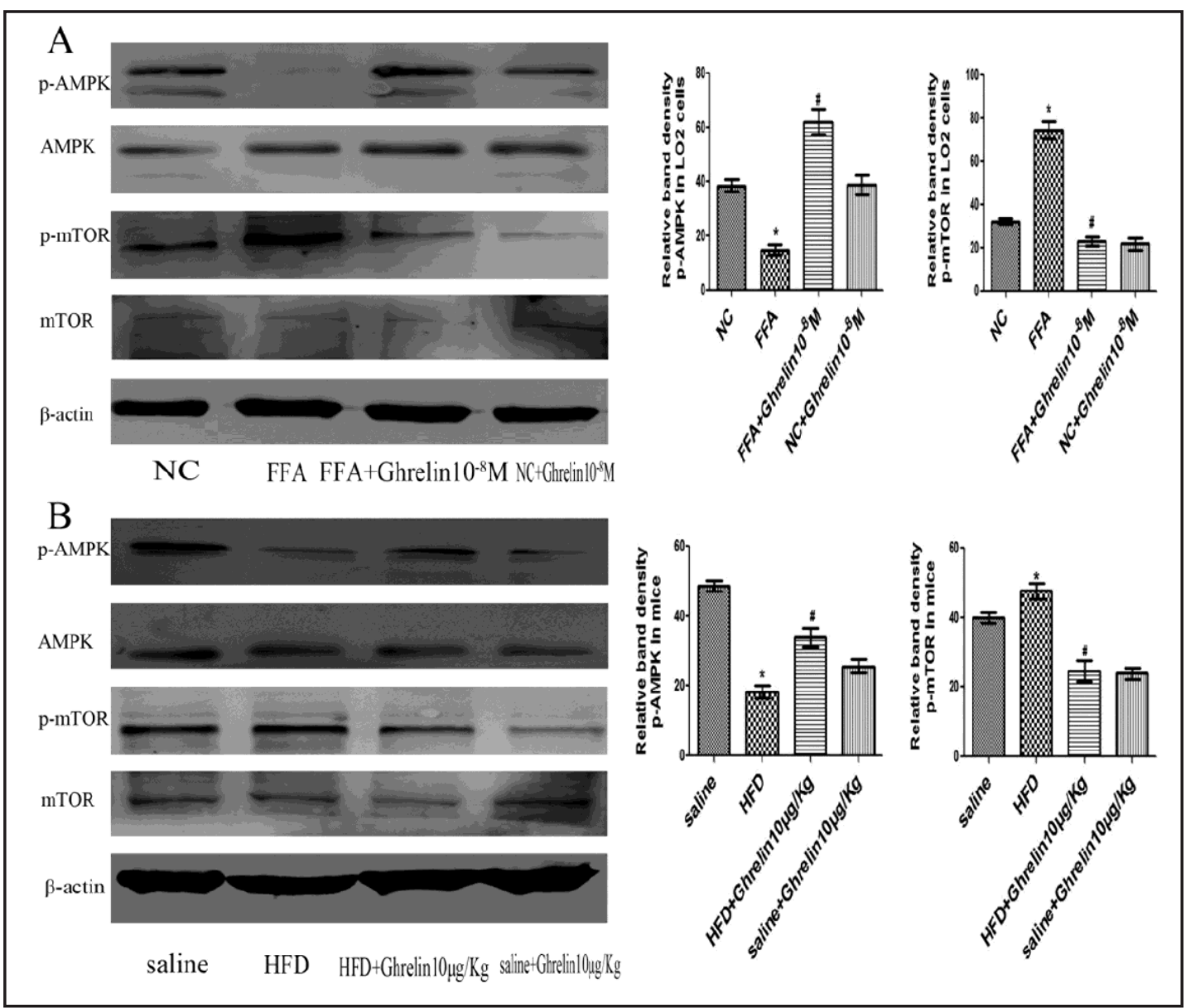

Fig. 5. Ghrelin promoted autophagy partly via restoration of AMPK/mTOR signaling pathway both in vitro and in vivo. (A and B) Western blots and quantitative evaluation of levels of p-AMPK, AMPK, p- mTOR and mTOR in LO2 cells and mice. ${ }^{*} P<0.05$ for FFA group versus NC group in cells, HFD group versus saline group in mice; \# $P<0.05$ versus FFA+ ghrelin group versus FFA group in cells, HFD+ ghrelin group versus HFD group in mice.

Ghrelin attenuated inflammatory injury by NF- $\kappa B$ inhibition and autophagy enhancement

To investigate the changes in NF- $\kappa$ B nuclear translocation, which is known as the activated condition, we extracted the cytoplasmic and nuclear proteins from each group and detected the protein level of NF- $\kappa$ B. In vitro, the FFA group had a low level of NF- $\kappa B$ in the cytoplasm but a high level in the nucleus. On the contrary, the FFA+ ghrelin group had a higher level of NF- $\kappa B$ in the cytoplasm and a lower level in the nucleus. Protein levels of I $\kappa B \alpha$ were reduced in the FFA group compared with the NC group, while ghrelin treatment ameliorated the decrease in IкB $\alpha$ protein compared with the FFA group (Fig. 6A). Similar results were obtained in mice experiment (Fig. 6B). Furthermore, imunofluorescence showed that FFA promoted NF- $\mathrm{KB}$ translocation in $\mathrm{LO} 2$ cells while ghrelin pretreatment reverse this process (Fig. 6C).

\section{Discussion}

NAFLD is being recognized increasingly as a major health burden [17]. It starts as a silent liver disease, but may progress into inflammation, fibrosis, and ultimately hepatoma. More importantly, NAFLD is closely associated with risk factors of coronary artery disease 


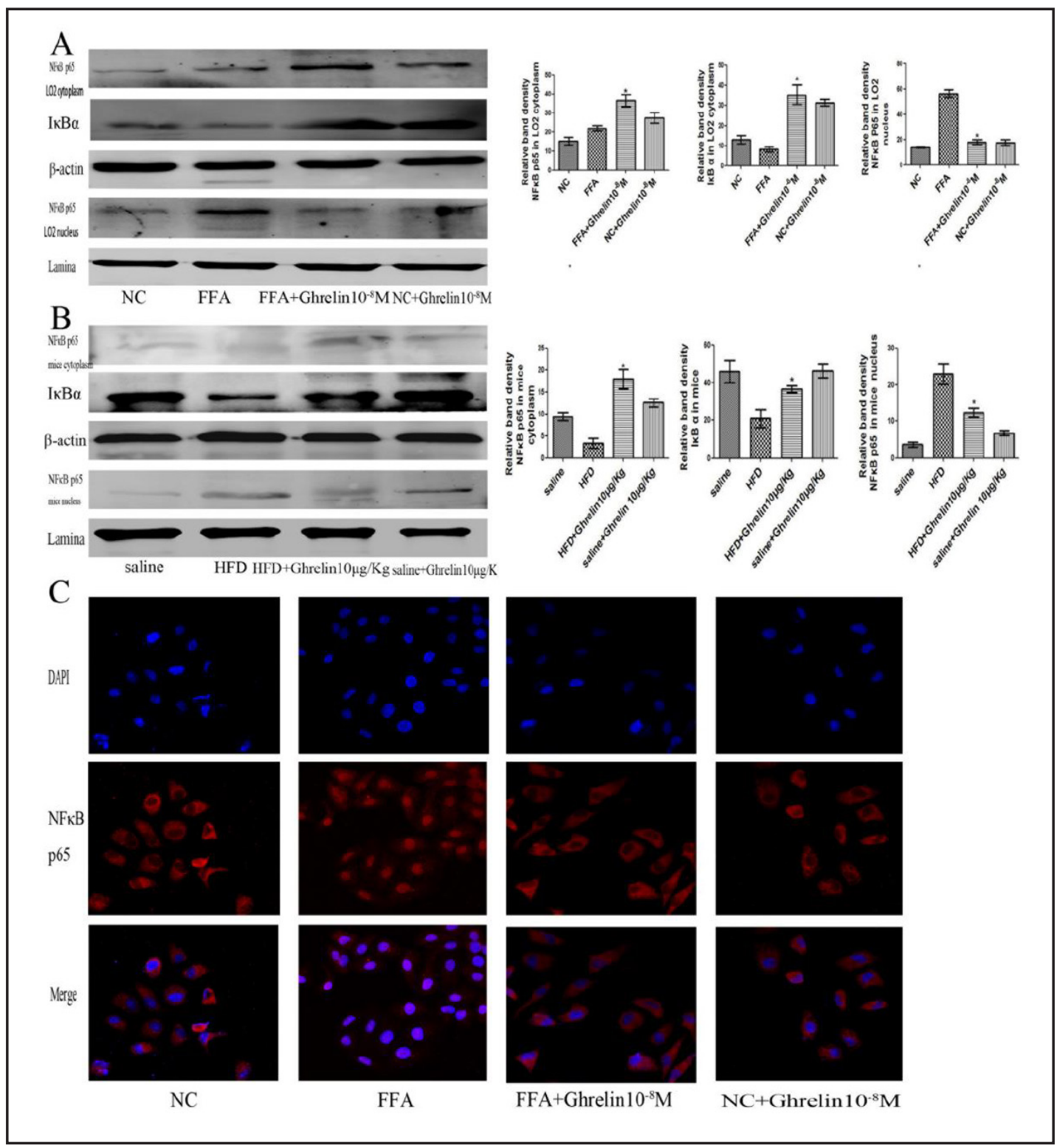

Fig. 6. Ghrelin administration attenuated inflammatory injury partly by NF- $\kappa \mathrm{B}$ p 65 inhibition both in vitro

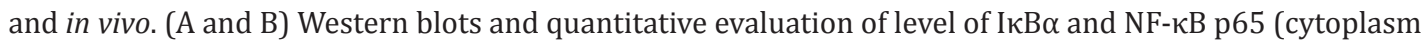
and nucleus, respectively) in vivo and in vitro. ${ }^{*} P<0.05$ for FFA+ ghrelin group versus FFA group in cells; HFD+ ghrelin group versus HFD group in mice. (C) Different distribution of NF- $\kappa$ B p65 in L02 cells, with or without ghrelin pretreatment before FFA stimulation, was evaluated by immunofluorescence.

[18]. However, effective methods to prevent and attenuate NAFLD are urgently needed.

In this study, we presented a novel drug ghrelin, which could play a role in NAFLD therapy. Ghrelin is a newly discovered peptide with various functions, including regulating energy metabolism, promoting cell proliferation [19]. Recent studies have demonstrated that ghrelin attenuates inflammatory injury $[13,15,16,20,21]$. Okamatsu et al. have shown that serum ghrelin level is significantly elevated with liver function index in overweight children [22]. Likewise, another study has reported that high levels of ghrelin are associated with low risk of developing fatty liver [23]. The most recent study has confirmed our belief that ghrelin could have a therapeutic effect in NAFLD [20]. However, the therapeutic mechanism of ghrelin in NAFLD remains largely unknown, especially the way to reduce lipid 


\section{Cellular Physiology Cell Physiol Biochem 2015;37:563-576 \begin{tabular}{l|l} 
and Biochemistry Published online: September 01, 2015 & $\begin{array}{l}\text { C) 2015 S. Karger AG, Basel } \\
\text { www.karger.com/cpb }\end{array}$ \\
\hline
\end{tabular} \\ Mao et al.: Ghrelin Reduced Lipotoxicity}

accumulation. Furthermore, the therapeutic effect of ghrelin has not been demonstrated in vitro. Our research is believed to be the first to demonstrate that ghrelin reduces lipid accumulation by enhancing autophagy, partly via restoration of the AMPK/mTOR signaling pathway.

We showed that ghrelin decreased intracellular lipids, with a concomitant increase in autophagy in vitro and in vivo. The dose at which we detected induction of autophagy in vitro was $10^{-8} \mathrm{M}$, as described previously [15]. The doses used in previous studies have been inconsistent. Therefore, we pretreated LO2 cells with a concentration gradient from $10^{-9}$ to $10^{-6} \mathrm{M}$ before stimulation with FFA mixture in the preliminary experiment. LC3II protein level and intracellular triglyceride level were detected. However, we found that cells treated with $10^{-8} \mathrm{M}$ ghrelin had the highest level of LC3II and lowest level of lipid (data not shown). We speculate that the effect of lipid reduction and autophagy was related to but not entirely concentration dependent. Whether ghrelin induces or inhibits autophagy is controversial. Wang et al. have shown that ghrelin inhibits doxorubicin cardiotoxicity by inhibiting excessive autophagy [24], which was contradicted with our research. We consider that the opposite effect of autophagy may be attributed to cell type, intervening measure and ghrelin concentration. Similarly, two doses of ghrelin $(10 \mathrm{ng} / \mathrm{kg}$ and $10 \mu \mathrm{g} / \mathrm{kg})$ were used in HFD mice, and $10 \mu \mathrm{g} / \mathrm{kg}$ showed a better therapeutic effect.

We observed a mild increase of LC3 protein level in HFD groups in vivo and FFA group in vitro, while ghrelin co-treatment significantly increased it. Short-term feeding with HFD in mice and FFA treatment in vitro promote autophagy as a self-protective mechanism to digest lipid partly via the mTOR complex, whereas long-term HFD inhibits autophagy by inhibition of the constitutive nuclear form of forkhead box 01, leading to acceleration of lipid accumulation [25]. Autophagy reduces lipids in NAFLD $[9,11]$ and in alcoholic fatty liver [26]. Triglyceride and cholesterol are taken up by autophagosomes and subsequently delivered to lysosomes for degradation into FFA, in a process known as lipophagy. Impaired lipophagy leads to excessive lipid accumulation [27]. Autophagy induction was accompanied with decreased intracellular lipid accumulation in our study. The ghrelin co-treated group had a significantly lower liver/body weight ratio and lipid accumulation in vivo. Furthermore, an autophagy inhibitor and stimulator (3-MA and rapamycin, respectively) were used to demonstrate that autophagy is essential for reduction of intracellular fat accumulation. As expected, 3-MA inhibited autophagy and increased triglyceride level. On the contrary, rapamycin lead to autophagy induction and obvious reduction of triglyceride level.

The AMPK signaling pathway plays a key role in the regulation of energy control. In starvation, AMPK is activated in response to an increase in AMP/ATP ratio, which requires phosphorylation of threonine 172 by an upstream kinase. AMPK is involved in the regulation of autophagy by inhibiting mTOR phosphorylation [28]. AMPK is inhibited by endoplasmic reticulum stress [29], oxidative stress and fat accumulation in NAFLD [14]. Furthermore, the effect of AMPK activation is stimulation of hepatic fatty acid oxidation and ketogenesis, inhibition of cholesterol synthesis, lipogenesis, and triglyceride synthesis [30], exerting an therapeutic effect on NAFLD. Ghrelin increases food intake and activates the AMPK signaling pathway $[14,20,31]$. Consistently, our study found that phosphorylation level of AMPK was reduced in the NAFLD group but restored by ghrelin without disturbing total AMPK. Meanwhile, p-mTOR was inhibited in the ghrelin co-treated group. Therefore, we speculate that ghrelin, an important energy regulator, stimulates appetite and activates AMPK, leading to the inhibition of p-mTOR and autophagy induction. NF- $\mathrm{kB}$ signaling pathway is a classic pathway in inflammatory reaction which is well established on the way from fat

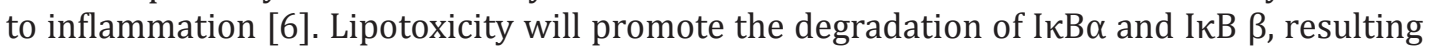
in the translocation of NF- $\mathrm{BB}$ into nucleus and subsequently the secretion of inflammatory cytokines. The anti-inflammatory effects of ghrelin are partly attributed to its NF- $\mathrm{B}$ inhibition. We showed that the I $\mathrm{B} \alpha \alpha$ levels in the ghrelin co-treated group were markedly higher than those in the HFD in vivo or FFA group in vitro. While NF- $\mathrm{BB}$ p65 was decreased in the HFD or FFA group but reversed in the cytoplasm in the ghrelin co-treated group. This demonstrates that ghrelin blocks I $\kappa \mathrm{B} \alpha$ degradation and prevents translocation of NF- $\kappa \mathrm{B}$ 


\section{Cellular Physiology Cell Physiol Biochem 2015;37:563-576 \begin{tabular}{l|l|l}
\hline DOI: 10.1159/000430377 & (C) 2015 S. Karger AG, Basel
\end{tabular} and Biochemistry Published online: September 01, 2015 \\ Mao et al.: Ghrelin Reduced Lipotoxicity}

into the nucleus.Autophagy induction may be another way to explain the anti-inflammatory effect of ghrelin on lipotoxicity. Appropriate autophagy is regarded as a cellular survival mechanism by degrading excessive inflammasomes and attenuating oxidative and ER stress.

Autophagy activates hepatic stellate cells [32], which leads to liver fibrosis. We have to take into account that while ghrelin may attenuate NAFLD-induced liver injury and reduce lipid accumulation in the short term, its long-term effect in fibrogenic cells may worsen chronic liver disease. However, another study demonstrated that ghrelin ameliorated hepatic fibrosis in rodents by attenuating hepatocyte injury [33]. Whether autophagy is involved in this process needs further study. We recognize that there were some limitations to our study. We did not detect the oxidation reaction products of cells or liver tissues. Lipid dye used in the immunofluorescence study may help to show better the relationship between autophagy and lipid droplets. More strategies of autophagy inhibition such as gene knockout may be more compelling.

In conclusion, our research first demonstrates that ghrelin attenuated NAFLD-induced liver injury by autophagy induction via restoration of the AMPK/mTOR signaling pathway and NF- $\kappa \mathrm{B}$ inhibition. Energy regulators such as ghrelin offer further insight into developing drugs for the prevention and treatment of NAFLD.

\section{Acknowledgements}

This work was supported by Research Grant for Key Clinical Discipline Construction of Shanghai Municipality, China, No. ZK2012B20.

\section{Disclosure Statement}

The authors declare no conflict of interests.

\section{References}

1 Williams CD, Stengel J, Asike MI, Torres DM, Shaw J, Contreras M, Landt CL, Harrison SA: Prevalence of nonalcoholic fatty liver disease and nonalcoholic steatohepatitis among a largely middle-aged population utilizing ultrasound and liver biopsy: a prospective study. Gastroenterology 2011;140:124-131.

2 Day CP, James OF: Steatohepatitis: a tale of two „hits“? Gastroenterology 1998;114:842-845.

3 Kawano Y, Cohen DE: Mechanisms of hepatic triglyceride accumulation in non-alcoholic fatty liver disease. J Gastroenterol 2013;48:434-441.

$4 \quad$ Natarajan SK, Ingham SA, Mohr AM, Wehrkamp CJ, Ray A, Roy S, Cazanave SC, Phillippi MA, Mott JL: Saturated free fatty acids induce cholangiocyte lipoapoptosis. Hepatology 2014;60:1942-1956.

5 Feng YY, Xu XQ Ji CB, Shi CM, Guo XR, Fu JF: Aberrant hepatic microRNA expression in nonalcoholic fatty liver disease. Cell Physiol Biochem 2014;34:1983-1997.

6 Day CP: From fat to inflammation. Gastroenterology 206;130:207-210.

7 Cai D, Yuan M, Frantz DF, Melendez PA, Hansen L, Lee J, Shoelson SE: Local and systemic insulin resistance resulting from hepatic activation of IKK-beta and NF-kappaB. Nat Med 2005;11:183-190.

8 Shintani T, Klionsky DJ: Autophagy in health and disease: a double-edged sword. Science 2004;306:990995.

9 Singh R, Kaushik S, Wang Y, Xiang Y, Novak I, Komatsu M, Tanaka K, Cuervo AM, Czaja MJ: Autophagy regulates lipid metabolism. Nature 2009;458:1131-1135.

10 10.Singh R: Autophagy and regulation of lipid metabolism. Results Probl Cell Differ 2010;52:35-46.

11 Sinha RA, Farah BL, Singh BK, Siddique MM, Li Y, Wu Y, Ilkayeva OR, Gooding J, Ching J, Zhou J, Martinez L, Xie S, Bay BH, Summers SA, Newgard CB, Yen PM: Caffeine stimulates hepatic lipid metabolism by the autophagy-lysosomal pathway in mice. Hepatology 2014;59:1366-1380.

12 Kojima M, Hosoda H, Date Y, Nakazato M, Matsuo H, Kangawa K: Ghrelin is a growth-hormone-releasing acylated peptide from stomach. Nature 1999;402:656-660. 


\section{Cellular Physiology Cell Physiol Biochem 2015;37:563-576 \begin{tabular}{l|l|l} 
DOI: 10.1159/000430377 & C 2015 S. Karger AG, Basel
\end{tabular} and Biochemistry Published online: September 01, 2015 \\ Mao et al.: Ghrelin Reduced Lipotoxicity}

13 Zhou X, Xue C: Ghrelin inhibits the development of acute pancreatitis and nuclear factor kappaB activation in pancreas and liver. Pancreas 2009;38:752-757.

14 Li Y, Hai J, Li L, Chen X, Peng H, Cao M, Zhang Q: Administration of ghrelin improves inflammation, oxidative stress, and apoptosis during and after non-alcoholic fatty liver disease development. Endocrine 2013;43:376-386.

15 Tong XX, Wu D, Wang X, Chen HL, Chen JX, Wang XX, Wang XL, Gan L, Guo ZY, Shi GX, Zhang YZ, Jiang W: Ghrelin protects against cobalt chloride-induced hypoxic injury in cardiac H9c2 cells by inhibiting oxidative stress and inducing autophagy. Peptides 2012;38:217-227.

16 Wang X, Wang XL, Chen HL, Wu D, Chen JX, Wang XX, Li RL, He JH, Mo L, Cen X, Wei YQ, Jiang W: Ghrelin inhibits doxorubicin cardiotoxicity by inhibiting excessive autophagy through AMPK and p38-MAPK. Biochem Pharmacol 2014;88:334-350.

17 Neuschwander-Tetri BA, Caldwell SH: Nonalcoholic steatohepatitis: summary of an AASLD Single Topic Conference. Hepatology 2003;37:1202-1219.

18 Kim D, Choi SY, Park EH, Lee W, Kang JH, Kim W, Kim YJ, Yoon JH, Jeong SH, Lee DH, Lee HS, Larson J, Therneau TM, Kim WR: Nonalcoholic fatty liver disease is associated with coronary artery calcification. Hepatology 2012;56:605-613.

19 Dixit VD, Schaffer EM, Pyle RS, Collins GD, Sakthivel SK, Palaniappan R, Lillard JW Jr, Taub DD: Ghrelin inhibits leptin- and activation-induced proinflammatory cytokine expression by human monocytes and $\mathrm{T}$ cells. J Clin Invest 2004;114:57-66.

20 Barazzoni R, Semolic A, Cattin MR, Zanetti M, Guarnieri G: Acylated ghrelin limits fat accumulation and improves redox state and inflammation markers in the liver of high-fat-fed rats. Obesity (Silver Spring) 2014;22:170-177.

21 Hou Y, An J, Hu XR, Sun BB, Lin J, Xu D, Wang T, Wen FQ: Ghrelin inhibits interleukin-8 production induced by hydrogen peroxide in A549 cells via NF-kappaB pathway. Int Immunopharmacol 2009;9:120-126.

22 Okamatsu Y, Matsuda K, Hiramoto I, Tani H, Kimura K, Yada Y, Kakuma T, Higuchi S, Kojima M, Matsuishi T: Ghrelin and leptin modulate immunity and liver function in overweight children. Pediatr Int 2009;51:9-13.

23 Gutierrez-Grobe Y, Villalobos-Blasquez I, Sánchez-Lara K, Villa AR, Ponciano-Rodríguez G, Ramos MH, Chavez-Tapia NC, Uribe M, Méndez-Sánchez N: High ghrelin and obestatin levels and low risk of developing fatty liver. Ann Hepatol 2010;9:52-57.

24 Wang X, Wang XL, Chen HL, Wu D, Chen JX, Wang XX, Li RL, He JH, Mo L, Cen X, Wei YQ, Jiang W: Ghrelin inhibits doxorubicin cardiotoxicity by inhibiting excessive autophagy through AMPK and p38-MAPK. Biochem Pharmacol 2014;88:334-350.

25 Liu HY, Han J, Cao SY, Hong T, Zhuo D, Shi J, Liu Z, Cao W: Hepatic autophagy is suppressed in the presence of insulin resistance and hyperinsulinemia: inhibition of Fox01-dependent expression of key autophagy genes by insulin. J Biol Chem 2009;284:31484-31492.

26 Ding WX, Li M, Chen X, Ni HM, Lin CW, Gao W, Lu B, Stolz DB, Clemens DL, Yin XM: Autophagy reduces acute ethanol-induced hepatotoxicity and steatosis in mice. Gastroenterology 2010;139:1740-1752.

27 Liu K, Czaja MJ: Regulation of lipid stores and metabolism by lipophagy. Cell Death Differ 2013;20:3-11.

28 Yin XM, Ding WX, Gao W: Autophagy in the liver. Hepatology 2008;47:1773-1785.

29 Zaouali MA, Boncompagni E, Reiter RJ, Bejaoui M, Freitas I, Pantazi E, Folch-Puy E, Abdennebi HB, GarciaGil FA, Roselló-Catafau J: AMPK involvement in endoplasmic reticulum stress and autophagy modulation after fatty liver graft preservation: a role for melatonin and trimetazidine cocktail. J Pineal Res 2013;55:6578.

30 Winder WW, Hardie DG: AMP-activated protein kinase, a metabolic master switch: possible roles in type 2 diabetes. Am J Physiol 1999;277:E1-10.

31 Carling D: AMP-activated protein kinase: balancing the scales. Biochimie 2005; 87:87-91.

32 Hernández-Gea V, Ghiassi-Nejad Z, Rozenfeld R, Gordon R, Fiel MI, Yue Z, Czaja MJ, Friedman SL: Autophagy releases lipid that promotes fibrogenesis by activated hepatic stellate cells in mice and in human tissues. Gastroenterology 2012;142:938-946.

33 Moreno M, Chaves JF, Sancho-Bru P, Ramalho F, Ramalho LN, Mansego ML, Ivorra C, Dominguez M, Conde L, Millán C, Marí M, Colmenero J, Lozano JJ, Jares P, Vidal J, Forns X, Arroyo V, Caballería J, Ginès P, Bataller R: Ghrelin attenuates hepatocellular injury and liver fibrogenesis in rodents and influences fibrosis progression in humans. Hepatology 2010;51:974-985. 